\title{
Diet and lifestyle triggers for gastro-oesophageal reflux disease: symptom identification
}

\author{
K. L. Oliver ${ }^{1}$, G. J. Davies ${ }^{1}$ and P. W. Dettmar ${ }^{2}$ \\ ${ }^{1}$ Nutrition Research Centre, London South Bank University, London SE1 OAA, UK and ${ }^{2}$ Technostics, The Deep Business \\ Centre, Tower Street, Hull, East Yorkshire HU1 4BG, UK
}

Previous studies have determined that diet and lifestyle may trigger the onset of symptoms of gastro-oesophageal reflux disease ${ }^{(1,2)}$. It remains unclear as to how these triggers precipitate the onset of specific symptoms.

The aim of the present study was to investigate possible diet and lifestyle trigger factors in Caucasian patients with heartburn. Ethical approval was gained from the Bedfordshire Local Research Ethics Committee. Ten male patients of mean age 53.1 (range 43-64) years and twelve female patients of mean age 53.1 (range 31-63) years were recruited from a general practitioner surgery in Bedfordshire, UK. All participants completed a symptoms and triggers diary for seven consecutive days, indicating the time of symptom onset, the type of symptom, severity and associated trigger factors. Symptoms were categorised using the validated gastro-oesophageal reflux disease questionnaire, the GERD impact scale ${ }^{(3)}$. This scale is used in the primary-care setting to assess how frequently symptoms are reported and the impact of these symptoms on patient's daily lifestyle.

As there were no significant differences between the genders they were analysed together.

\begin{tabular}{lcc}
\hline & \multicolumn{2}{c}{ GERD impact scale symptom } \\
& Pain or burning sensation & \\
\cline { 2 - 3 } Trigger factor & $\%(n$ 22) & $n$ \\
\hline Fatty foods & 36 & 8 \\
Stress & 36 & 8 \\
Alcohol & 32 & 7 \\
Eating quickly & 27 & 6 \\
Large meal & 27 & 6 \\
Bending down & 23 & 5 \\
Spicy foods & 23 & 5 \\
Time of eating & 18 & 4 \\
Chocolate & 14 & 3 \\
Citrus fruits & 14 & 3 \\
Eating whilst slouching & 14 & 3 \\
Lying down & 14 & 3 \\
Coffee & 9 & 2 \\
Sitting & 9 & 2 \\
Sleeping on right side & 9 & 2 \\
Smoking & 5 & 1 \\
\hline
\end{tabular}

\begin{tabular}{lcc}
\hline & \multicolumn{2}{c}{$\begin{array}{c}\text { GERD impact scale symptom } \\
\text { Other acid-related symptoms }\end{array}$} \\
\cline { 2 - 3 } Trigger factor & $\%(n$ 22) & $n$ \\
\hline Spicy foods & 27 & 6 \\
Large meal & 23 & 5 \\
Time of eating & 23 & 5 \\
Stress & 18 & 4 \\
Citrus fruits & 14 & 3 \\
Coffee & 14 & 3 \\
Lying down & 14 & 3 \\
Sitting & 14 & 3 \\
Smoking & 14 & 3 \\
Alcohol & 9 & 2 \\
Bending down & 9 & 2 \\
Eating quickly & 9 & 2 \\
Fatty foods & 9 & 2 \\
Chocolate & 5 & 1 \\
Eating whilst slouching & 5 & 1 \\
Sleeping on right side & 5 & 1 \\
\hline
\end{tabular}

The results suggest that there are many trigger factors associated with symptoms of gastro-oesophageal reflux disease. It can be identified that there are different symptoms reported in response to certain trigger factors. Fatty foods and stress were the most reported to precipitate the onset of pain or burning in the chest area. In comparison, spicy foods was the most reported trigger factor for other acidrelated symptoms, which includes acid regurgitation, pain or burning in the upper stomach and sore throat. In light of these interesting findings further investigation is needed to gain a better understanding of these diet and lifestyle trigger factors and their role in the onset and manifestation of symptoms.

1. Nilsson M, Johnsen R, Ye W et al. (2004) Gut 53, 1730-1735.

2. El-Serag HB, Satia JA \& Rabeneck L (2005) Gut 54, 11-17.

3. Jones R, Coyne K \& Wiklund I (2007) Aliment Pharmacol Ther 25, 1451-1459. 